\title{
Analysis of Mesh Router Placement in Wireless Mesh Networks Using Friedman Test Considering Different Meta-heuristics
}

\begin{abstract}
:
In this paper, we deal with connectivity and coverage problem in Wireless Mesh Networks (WMNs). We used Friedman test to compare Genetic Algorithm (GA), Tabu Search (TS), Hill Climbing (HC) and Simulated Annealing (SA). We found out that GA, TS, HC and SA have differences in their performance. Then, we used the implemented systems WMN-GA, WMN-TS, WMN-HC and WMN-SA to evaluate and compare the performance of the systems for different distributions of mesh clients in terms of Size of Giant Component (SGC) and Number of Covered Mesh Clients (NCMC). The simulation results show that for Uniform distribution the WMN-HC and WMN-SA perform better than WMNGA and WMN-TS. However, for small radius of communication distance, the SGC of WMN-TS is better than other systems. For Normal distribution, for big radius of communication distance, the WMN-GA has the best performance. For Exponential distribution, the WMN-HC and WMN-SA perform better than WMN-GA for all communication distances. For Weibull distribution, the WMNTS has a good performance for small radius of communication distance, but for big radius of communication distances the WMN-GA, WMN-HC and WMN-SA perform better.

Keywords: Wireless Mesh Networks; Friedman Test; Genetic Algorithm; Tabu Search Algorithm; Hill Climbing Algorithm; Simulated Annealing Algorithm; Connectivity; Coverage.
\end{abstract}

\section{Introduction}

The wireless networks and devices are becoming increasingly popular and they provide users access to information and communication anytime and anywhere (Boyinbode et. al., 2011), (Puzar et. al., 2011), (Martins et. al., 2011), (Koyama et. al., 2011), (Hiyama et. al., 2012), (Ikeda, 2012), Spaho et. al. (2011), (Aikebaier et. al., 2011), (Xhafa et. al., 2012), (Xhafa et. al., 2012), (Goto et. al., 2013). Wireless Mesh Networks (WMNs) (Akyildizet. al., 2005) are a subclass of wireless networks that are attracting a lot of research attention recently. WMNs are important networking infrastructures. These networks are made up of wireless nodes, organized in a mesh topology, where mesh routers are interconnected by wireless links and provide Internet connectivity to mesh clients.

WMNs distinguish for their low cost nature that makes them attractive for providing wireless Internet connectivity. Moreover, such infrastructure can be used to deploy community networks, metropolitan area networks, municipal and, corporative networks, and to support applications for urban areas, medical, transport and surveillance systems.

The main issue of WMNs is to achieve network connectivity and stability as well as QoS in terms of user coverage (Ernst et. al., 2013). This problem is very closely 
related to the family of node placement problems in WMNs (Muthaiah, 2008), (Tang, 2009), (Franklin et. al., 2007), (Vanhatupa et. al., 2007), among them, the mesh router mesh nodes placement. Here, we consider the version of the mesh router nodes placement problem in which we are given a grid area where to deploy a number of mesh router nodes and a number of mesh client nodes of fixed positions (of an arbitrary distribution) in the grid area. The objective is to find a location assignment for the mesh routers to the cells of the grid area that maximizes the network connectivity and client coverage. As node placement problems are known to be computationally hard to solve for most of the formulations (Garey et. al., 1979), (Lim et. al., 2005), (Wang et. al., 2007), Genetic Algorithms (GAs) and local search methods like Tabu Search (TS), Hill Climbing (HC) and Simulated Annealing (SA) have been recently investigated as effective resolution methods. However, GAs require the user to provide values for a number of parameters and a set of genetic operators to achieve the best GA performance for the problem (Yao, 1993), (Denzinger et. al., 2006), (Odetayo, 1997), (Xhafa et. al., 2008), (Xhafa et. al., 2007), (Xhafa et. al., 2009), (Oda et. al., 2012).

In this paper, we deal with connectivity and coverage problem of WMNs. First, we used Friedman test to check if we can compare GA, TS, HC and SA. Then, we used the web interfaces to evaluate and compare the performance of four systems for different distributions of mesh clients in terms of Size of Giant Component (SGC) and Number of Covered Mesh Clients (NCMC). The simulation results show that for Uniform distribution the WMN-HC and WMN-SA perform better than WMN-GA and WMN-TS. However, for small radius of communication distance, the SGC of WMN-TS is better than other systems. For Normal distribution, for big radius of communication distance, the WMNGA has the best performance. For Exponential distribution, the WMN-HC and WMN-SA perform better than WMN-GA for all communication distances. For Weibull distribution, the WMN-TS has a good performance for small radius of communication distance, but for big radius of communication distances the WMN-GA, WMN-HC and WMN-SA perform better.

The rest of the paper is organized as follows. The mesh router nodes placement problem is defined in Section 2. We give a brief introduction of GAs, TS, HC and SA algorithms and Web Interfaces in Section 3. The simulation results are given in Section 4. In Section 5, we give some conclusions and future work.

\section{Mesh Router Node Placement Problem}

In this problem, we are given a grid area arranged in cells where to distribute a number of mesh router nodes and a number of mesh client nodes of fixed positions (of an arbitrary distribution) in the grid area. The objective is to find a location assignment for the mesh routers to the cells of the grid area that maximizes the network connectivity and client coverage. Network connectivity is measured by the size of the giant component of the resulting WMN graph, while the user coverage is simply the number of mesh client nodes that fall within the radio coverage of at least one mesh router node.

An instance of the problem consists as follows.

- $N$ mesh router nodes, each having its own radio coverage, defining thus a vector of routers. 
- An area $W \times H$ where to distribute $N$ mesh routers. Positions of mesh routers are not pre-determined, and are to be computed.

- $M$ client mesh nodes located in arbitrary points of the considered area, defining a matrix of clients.

It should be noted that network connectivity and user coverage are among most important metrics in WMNs and directly affect the network performance. Nonetheless, network connectivity is usually considered as more important than user coverage.

Notice from the above definition that mesh client nodes can be arbitrarily situated in the given area. For evaluation purposes, it is, however, interesting to consider concrete distributions of mesh client nodes such as Uniform, Normal, Exponential and Weibull distributions.

In fact, we can formalize an instance of the problem by constructing an adjacency matrix of the WMN graph, whose nodes are router nodes and client nodes and whose edges are links between nodes in the mesh network. Each mesh node in the graph is a triple $v=\langle x, y, r>$ representing the 2D location point and $r$ is the radius of the transmission range. There is an arc between two nodes $u$ and $v$, if $v$ is within the transmission circular area of $u$. It should be noticed here that the deployment grid area is partitioned by cells, representing graph nodes, where we can locate mesh router nodes. We assume that in a cell, both a mesh router node and a mesh client node can be placed.

Optimization setting: For optimization problems having two or more objective functions, two settings are usually considered: the hierarchical and simultaneous optimization. In the former, the objectives are classified (sorted) according to their priority. Thus, for the bi-objective case, one of the objectives, say $f_{1}$, is considered as a primary objective and the other, say $f_{2}$, as secondary one. The meaning is that we first try to optimize $f_{1}$, and then when no further improvements are possible, we try to optimize $f_{2}$ without worsening the best value of $f_{2}$. In the case of WMNs, the hierarchical approach is used due achieving network connectivity is considered more important than user coverage. It should be noted that due to this optimization priority, some client nodes may not be covered due the user coverage is less optimized.

\section{Optimization Resolution Methods and Web Interfaces}

Purely random placements would produce poor performance due to far from optimal router placement. Therefore, using more efficient methods is crucial for node placement nodes in WMNs. Due to computational intractability of the problem, exact methods can only solve to optimality small size instances, and therefore heuristic and meta-heuristic approaches are the de facto approach to solve the problem for practical purposes.

\subsection{Population-based Method: Genetic Algorithms}

GAs have shown their usefulness for the resolution of many computationally hard combinatorial optimization problems. They are, of course, a strong candidate for efficiently solving mesh router nodes placement problem in WMNs. For the purpose of this work we have used the template given in Algorithm 1.

As can be seen from the template, several parameters intervene in the GAs: population size, intermediate population size, number of evolution steps, crossover probability, 


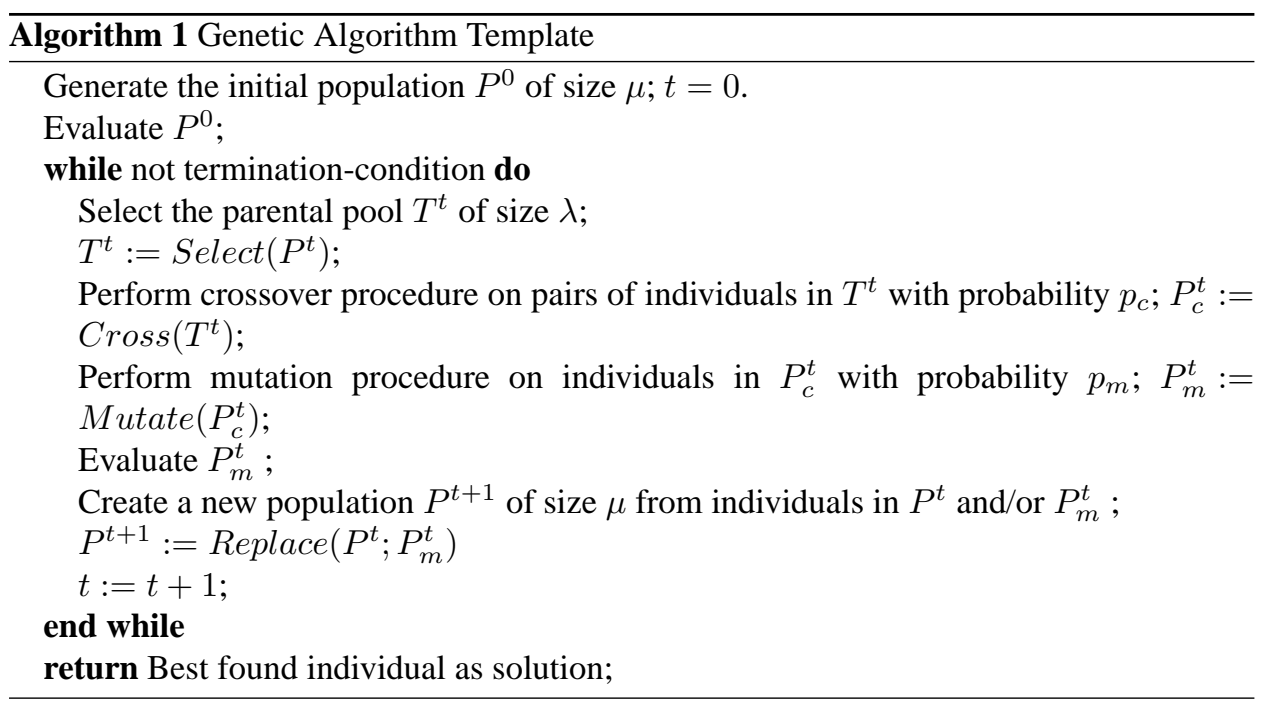

mutation probability and parameters for replacement strategies. On the other hand, there are the (families of) genetic operators: crossover operators, mutation operators, selection operators and replacement operators. As there are potentially large range values for parameters and different versions of operators, their tuning becomes crucial to the GA's performance.

\subsection{Local Search Method: Tabu Search Algorithm}

Tabu Search (TS) method was introduced by Glover (Glover, 1986) as a high-level algorithm that uses other specific heuristics to guide the search; the objective is to perform an intelligent exploration of the search space that would eventually allow to avoid getting trapped into local optima. The objective is thus to remedy one of the main issues of local search methods, namely the useless search in neighborhood of local optima without further improvements due to re-visiting solutions or paths of solutions already explored. This is achieved by giving the tabu status to solutions visited in the recent search. TS is also designed to be a flexible method, so that the tabu status of solutions can be waived, in case they have been prohibited for a long while or if they satisfy some aspiration criteria. The classification of some solutions as tabu is achieved through the intelligent use of adaptive memory, which is allowed to evolve and eventually change the status of tabu solutions. The main features of the TS method are that of adaptive memory and responsive exploration. Again, the adaptive memory is the basis to guide the search in taking intelligent decisions. This gives the TS method advantages with regard to other memoryless methods, being these local search methods (HC, SA, etc.) or population based methods (GAs, Memetic Algorithms, etc.). On the other hand, the responsive exploration enables the method to select some solutions which though not so good at the current search iteration might at long run lead to promising areas of good solutions in the search space (see Algorithm 2).

\subsection{Local Search Method: Hill Climbing Algorithm}

We present here the particularization of the HC algorithm (see Algorithm 3) for the mesh router node placement problem in WMNs. 

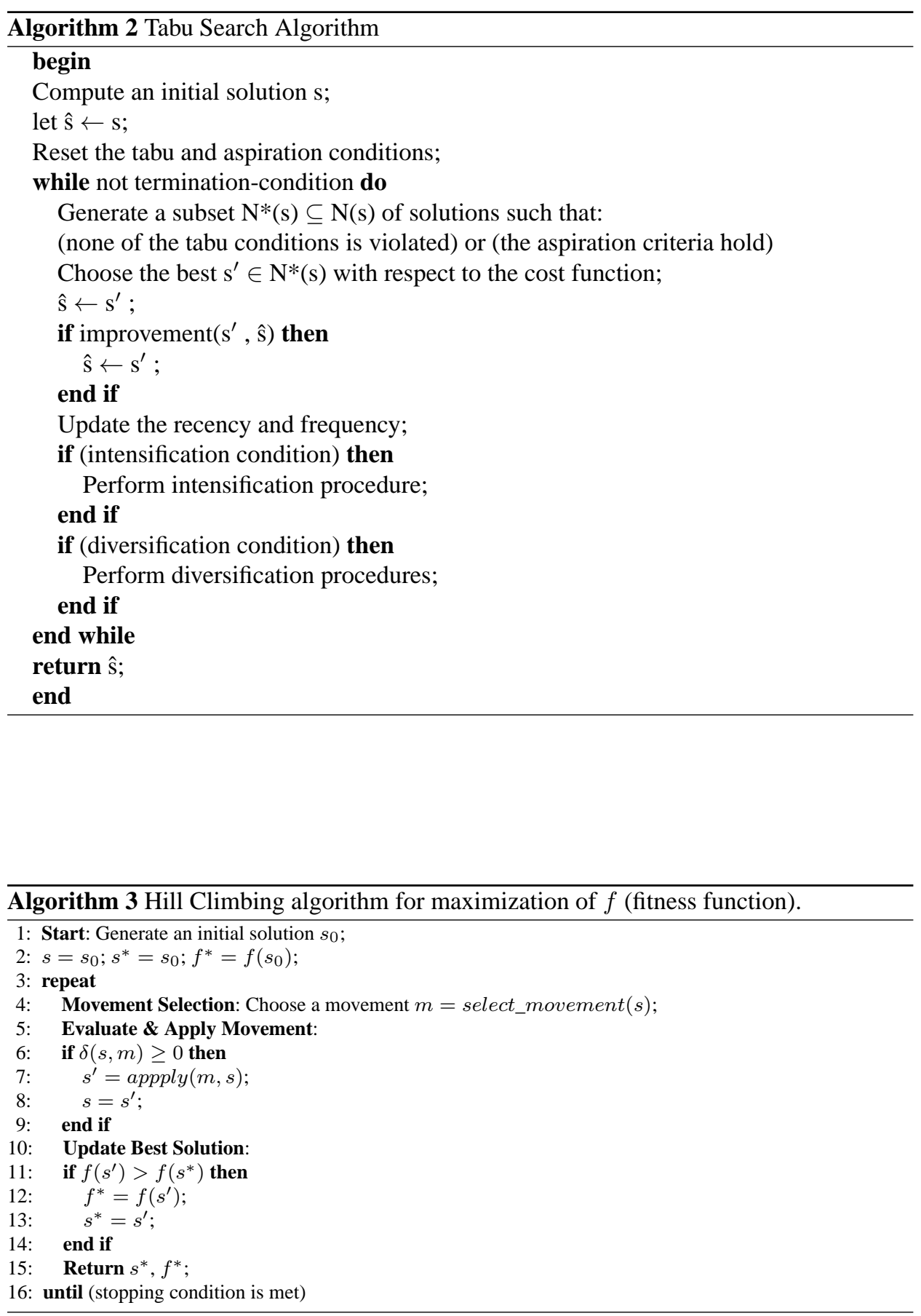


\section{Initial Solution}

The algorithms starts by generating an initial solution either random or by ad hoc methods (Xhafa et. al., 2009).

\section{Evaluation of fitness function}

An important aspect is the determination of an appropriate objective function and its encoding. In our case, the fitness function follows a hierarchical approach in which the main objective is to maximize the size of giant component in WMN.

\section{Neighbor selection and movement types}

The neighborhood $N(s)$ of a solution $s$ consists of all solutions that are accessible by a local move from $s$. We have considered three different types of movements. The first, called Random, consists in choosing a router at random in the grid area and placing it in a new position at random. The second move, called Radius, chooses the router of the largest radio and places it at the center of the most densely populated area of client mesh nodes (see Algorithm 4). Finally, the third move, called Swap, consists in swapping two routers: the one of the smallest radio situated in the most densely populated area of client mesh nodes with that of largest radio situated in the least densely populated area of client mesh nodes. The aim is that largest radio routers should serve to more clients by placing them in more dense areas.

We also considered the possibility to combine the above movements in sequences of movements. The idea is to see if the combination of these movements offers some improvement over the best of them alone. We called this type of movement Combination:

$<$ Rand $_{1}, \ldots$, Rand $_{k} ;$ Radius $_{1}, \ldots$, Radius $_{k} ;$

$S$ wap $_{1}, \ldots$, Swap $_{k}>$,

where $k$ is a user specified parameter.

\section{Acceptability Criteria}

The acceptability criteria for newly generated solution can be done in different ways (simple ascent, steepest ascent, or stochastic). In our case, we have adopted the simple ascent, that is, if $s$ is current solution and $m$ is a movement, the resulting solution $s^{\prime}$ obtained by applying $m$ to $s$ will be accepted, and hence become current solution, iff the fitness of $s^{\prime}$ is at least as good as fitness of solution $s$. In terms of $\delta$ function, $s^{\prime}$ is accepted and becomes current solution if $\delta(s, m) \geq 0$. It should be noted that in this definition we are also accepting solutions that have the same fitness as previous solution. The aim is to give chances to the search to move towards better solutions in solution space. A more strict version would be to accept only solutions that strictly improve the fitness function $(\delta(s, m)>0)$.

\subsection{Local Search Method: Simulated Annealing Algorithm}

The SA algorithm (Kirkpatrik et. al., 1983) is a generalization of the metropolis heuristic. Indeed, SA consists of a sequence of executions of metropolis with a progressive decrement of the temperature starting from a rather high temperature, where almost any move is accepted, to a low temperature, where the search resembles HC. In fact, it can be seen 


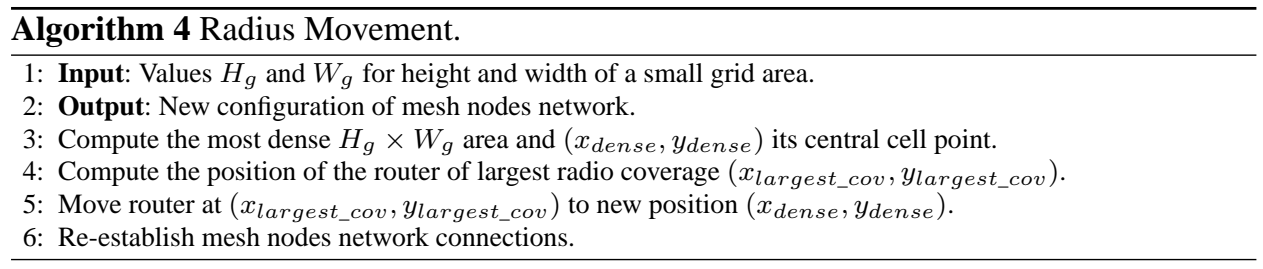

as a hill-climber with an internal mechanism to escape local optima (see pseudo-code in Algorithm 5). In SA, the solution $s^{\prime}$ is accepted as the new current solution if $\delta \leq 0$ holds, where $\delta=f\left(s^{\prime}\right)-f(s)$. To allow escaping from a local optimum, the movements that increase the energy function are accepted with a decreasing probability $\exp (-\delta / T)$ if $\delta>0$, where $T$ is a parameter called the "temperature". The decreasing values of $T$ are controlled by a cooling schedule, which specifies the temperature values at each stage of the algorithm, what represents an important decision for its application (a typical option is to use a proportional method, like $T_{k}=\alpha \cdot T_{k-1}$ ). SA usually gives better results in practice, but uses to be very slow. The most striking difficulty in applying SA is to choose and tune its parameters such as initial and final temperature, decrement of the temperature (cooling schedule), equilibrium detection, etc.

For further details on initial solution, fitness evaluation and movement types, refer to (Xhafa et. al., 2011). However, the acceptability criteria of neighboring solutions is now different, as explained next.

\section{Acceptability Criteria}

The acceptability criteria for newly generated solution is based on the definition of a threshold value (accepting threshold) as follows. We consider a succession $t_{k}$ such that $t_{k}>t_{k+1}, t_{k}>0$ and $t_{k}$ tends to 0 as $k$ tends to infinity. Then, for any two solutions $s_{i}$ and $s_{j}$, if fitness $\left(s_{j}\right)-$ fitness $\left(s_{i}\right)<t_{k}$, then accept solution $s_{j}$.

For the SA, $t_{k}$ values are taken as accepting threshold but the criterion for acceptance is probabilistic:

- If fitness $\left(s_{j}\right)$ - fitness $\left(s_{i}\right) \leq 0$ then $s_{j}$ is accepted.

- If fitness $\left(s_{j}\right)$-fitness $\left(s_{i}\right)>0$ then $s_{j}$ is accepted with probability $\exp \left[\left(\right.\right.$ fitness $\left(s_{j}\right)-$ fitness $\left.\left.\left(s_{i}\right)\right) / t_{k}\right] \quad$ (at iteration $k$ the algorithm generates a random number $R \in(0,1)$ and $s_{j}$ is accepted if $R<\exp \left[\left(\right.\right.$ fitness $\left(s_{j}\right)-$ fitness $\left.\left.\left.\left(s_{i}\right)\right) / t_{k}\right]\right)$.

In this case, each neighbor of a solution has a positive probability of replacing the current solution. The $t_{k}$ values are chosen in way that solutions with large increase in the cost of the solutions are less likely to be accepted (but there is still a positive probability of accepting them).

\subsection{Web Interface}

The Web application (Xhafa et. al., 2012) follows a standard Client-Server architecture and is implemented using LAMP (Linux + Apache + MySQL + PHP) technology (see Fig. 1). Remote users (clients) submit their requests by completing first the parameter setting. The parameter values to be provided by the user are classified into three groups, as follows. 

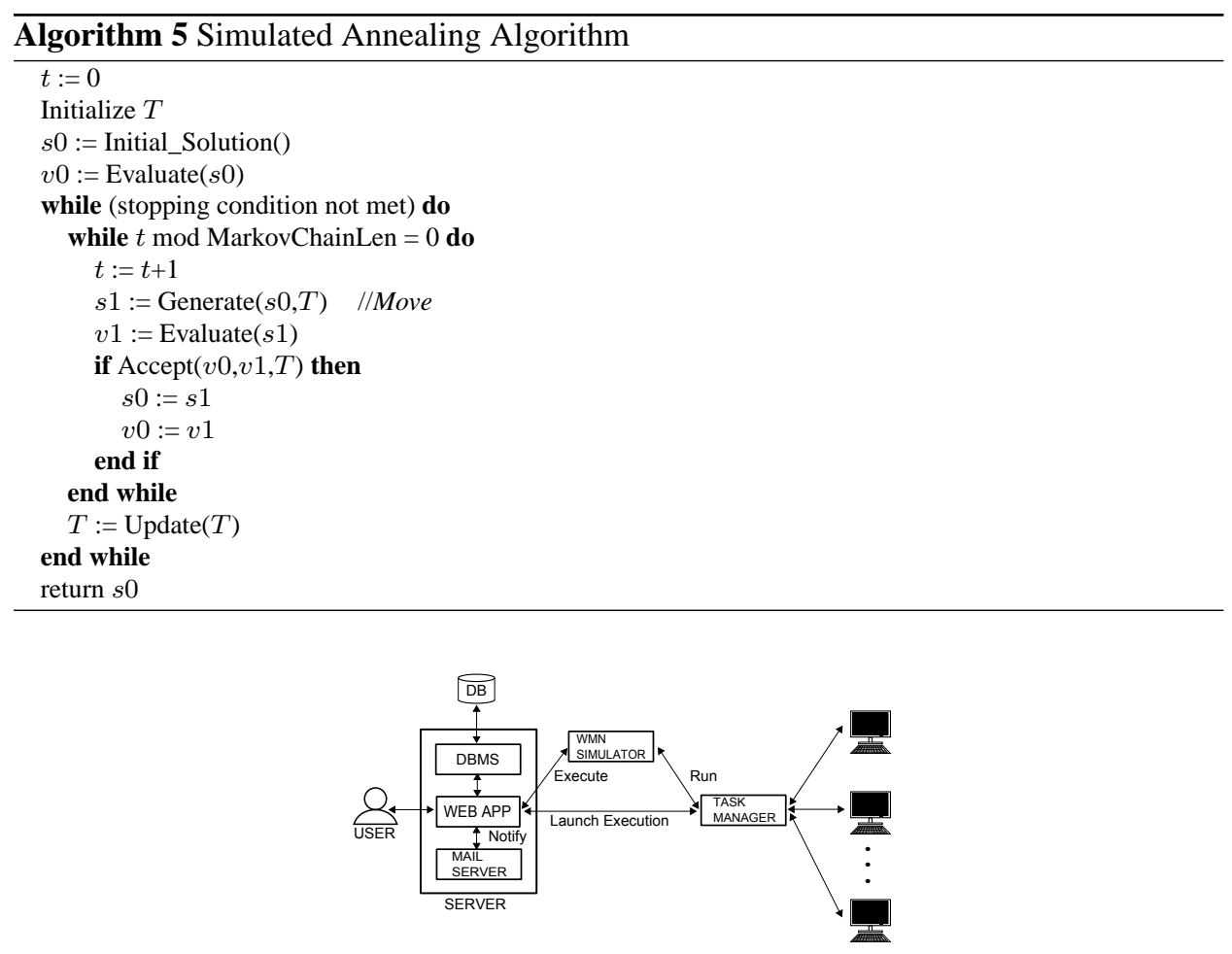

Figure 1 System structure for Web interface

- Parameters related to the problem instance: These include parameter values that determine a problem instance to be solved and consist of number of router nodes, number of mesh client nodes, client mesh distribution, radio coverage interval and size of the deployment area.

- Parameters of the resolution method: Each method has its own parameters. In Fig. 2 are shown the the GUI of Web Interfaces for the parameter setting of GA, TS, HC and SA.

- Execution parameters: These parameters are used for stopping condition of the resolution methods and include number of iterations and number of independent runs. The former is provided as a total number of iterations and depending on the method is also divided per phase (e.g., number of iterations in a exploration). The later is used to run the same configuration for the same problem instance and parameter configuration a certain number of times.

\section{Simulation Results}

The Friedman test Friedman (1937) is a nonparametric statistical test of multiple group measures. It can be used to approve the null hypothesis that the multiple group measures have the same variance to a certain required level of significance. On the other hand, 


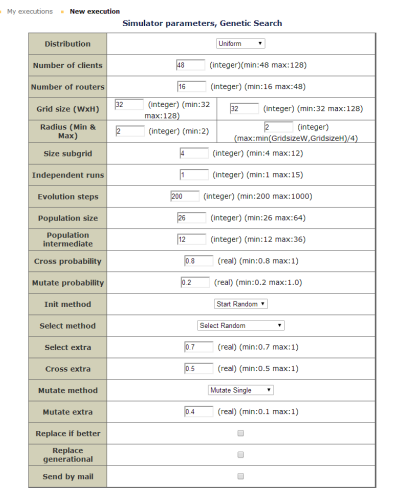

(a) $\mathrm{GA}$

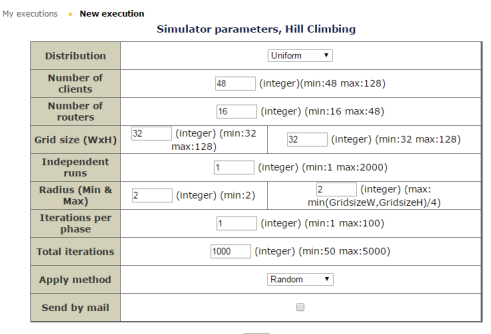

(c) $\mathrm{HC}^{\text {Rum }}$

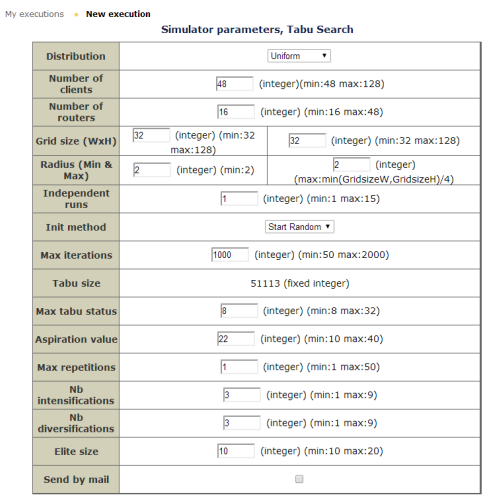

(b) TS

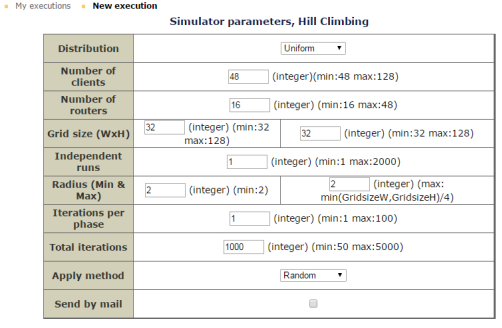

(d) SA

Figure 2 Web interfaces

Table 1 Input parameters of WMN-GA.

\begin{tabular}{c|c}
\hline Parameters & Values \\
\hline \hline Number of clients & 48 \\
Number of routers & 16 \\
Grid width & 32 [units] \\
Grid height & 32 [units] \\
Communication Distance (min:max) & $2 \times 2: \mathrm{n} \times \mathrm{n}(\mathrm{n}=2,4,6,8)$ [units] \\
Independent runs & 10 \\
Initial Router Placement Method & HotSpot \\
Number of Generations & 200 \\
Population size & 32 \\
Selection Method & Linear Ranking \\
Crossover rate & $80 \%$ \\
Mutate Method & Single \\
Mutate rate & $20 \%$ \\
Distribution of Clients & $\mathrm{N}, \mathrm{U}, \mathrm{E}, \mathrm{W}$ \\
\hline
\end{tabular}

failing to approve the null hypothesis shows that they have different variance values. We analyze the difference in performance between GA, TS, HC and SA using Friedman test in MATLAB. We considered as null hypothesis $H_{0}$ that there is difference in the performance between GA, TS, HC and SA. As alternative hypothesis we considered $H_{1}$ that there is no difference in the performance of GA, TS, HC and SA. As value of the hypothesis testing we took the maximum value of number of covered mesh clients and size of giant component. The significance level in this testing hypothesis is $\alpha=0.05$. We reject $H_{0}$ for $p>\alpha$ (p-value is the probability of obtaining a test statistic at least as extreme as the one that was actually observed, assuming that the null hypothesis is true). Further, since there is a correspondence between GA, TS, HC and SA, we used Friedman test. The results 
Table 2 Input parameters of WMN-TS.

\begin{tabular}{c|c}
\hline Parameters & Values \\
\hline \hline Number of clients & 48 \\
Number of routers & 16 \\
Grid width & $32[\mathrm{units}]$ \\
Grid height & $32[\mathrm{units}]$ \\
Communication Distance (min:max) & $2 \times 2: \mathrm{n} \times \mathrm{n}(\mathrm{n}=2,4,6,8)$ [units] \\
Independent runs & 10 \\
Initial Router Placement Method & HotSpot \\
Max Iterations & 2000 \\
Max Tabu Status & 9 \\
Aspiration Value & 15 \\
Max Repetitions & 15 \\
Number of Intensifications & 4 \\
Number of Diversifications & 4 \\
Elite Size & 10 \\
Distribution of Clients & $\mathrm{N}, \mathrm{U}, \mathrm{E}, \mathrm{W}$ \\
\hline
\end{tabular}

Table 3 Input parameters of WMN-HC.

\begin{tabular}{c|c}
\hline Parameters & Values \\
\hline \hline Number of clients & 48 \\
Number of routers & 16 \\
Grid width & 32 [units] \\
Grid height & 32 [units] \\
Communication Distance (min:max) & $2 \times 2: \mathrm{n} \times \mathrm{n}(\mathrm{n}=2,4,6,8)$ [units] \\
Independent runs & 10 \\
Initial Router Placement Method & HotSpot \\
Iteration per Phases & 9 \\
Total Iterations & 2000 \\
Replacement Method & Combination \\
Distribution of Clients & N, U, E, W \\
\hline
\end{tabular}

Table 4 Input parameters of WMN-SA.

\begin{tabular}{c|c}
\hline Parameters & Values \\
\hline \hline Number of clients & 48 \\
Number of routers & 16 \\
Grid width & 32 [units] \\
Grid height & 32 [units] \\
Communication Distance (min:max) & $2 \times 2: \mathrm{n} \times \mathrm{n}$ (n=2, 4, 6, 8) [units] \\
Independent runs & 10 \\
Initial Router Placement Method & HotSpot \\
Iteration per Phases & 9 \\
Total Iterations & 2000 \\
Temperature & 1 \\
Replacement Method & Combination \\
Distribution of Clients & $\mathrm{N}, \mathrm{U}, \mathrm{E}, \mathrm{W}$ \\
\hline
\end{tabular}

Table 5 The p-value of SGC of Friedman test.

\begin{tabular}{c|c|c|c|c}
\hline & GA & TS & HC & SA \\
\hline \hline GA & N/A & 0.0027 & 0.0027 & 0.0027 \\
TS & 0.0027 & N/A & 0.3173 & 0.3173 \\
HC & 0.0027 & 0.3173 & N/A & 0.3173 \\
SA & 0.0027 & 0.3173 & 0.3173 & N/A \\
\hline
\end{tabular}

of p-values for Friedman test for SGC are shown in Table 5 and $H_{1}$ is rejected because $p<0.05$. In this case, we adopted $H_{1}$. For NCMC, the p-values are shown in Table 6, and we adopt $H_{1}$ since $p<0.05$.

In this work, we consider different radius of communication distances and evaluate the performance of WMN-GA, WMN-TS, WMN-HC and WMN-SA for Uniform (U), Normal 
10.

Table 6 The p-value of NCMC of Friedman test.

\begin{tabular}{c|c|c|c|c}
\hline & GA & TS & HC & SA \\
\hline \hline GA & N/A & 0.6171 & 0.0334 & 0.0334 \\
TS & 0.6171 & N/A & 0.0196 & 0.0196 \\
HC & 0.0334 & 0.3173 & N/A & 0.1797 \\
SA & 0.0334 & 0.0196 & 0.1797 & N/A \\
\hline
\end{tabular}

$(\mathrm{N})$, Exponential (E) and Weibull (W) distributions. The number of mesh routers for all scenarios is considered 16 and the number of mesh clients 48 . The input parameters for WMN-GA, WMN-TS, WMN-HC and WMN-SA are shown in Table 1, Table 2, Table 3 and Table 4, respectively.

In Fig. 3, Fig. 4, Fig. 5 and Fig. 6 are shown simulation results for Uniform distribution using WMN-GA, WMN-TS, WMN-HC and WMN-SA, respectively. We used bar graph representation that shows the minimum, average and maximum value. We also show the visualization of nodes after the placement. If we compare the results, the WMN$\mathrm{HC}$ and WMN-SA perform better than WMN-GA and WMN-TS. However, for radius of communication distance $2 \times 2: 2 \times 2$, the SGC of WMN-TS is better than other systems.

In Fig. 7, Fig. 8, Fig. 9 and Fig. 10 are shown simulation results for Normal distribution using WMN-GA, WMN-TS, WMN-HC and WMN-SA, respectively. If we compare the results, the WMN-HC and WMN-SA perform better than WMN-GA and WMN-TS. But, for radius of communication distance $2 \times 2: 8 \times 8$, the SGC and NCMC (see Fig. 7(a) and Fig. 7(b)) of WMN-GA are better than other systems.

In Fig. 11, Fig. 12, Fig. 13 and Fig. 14 are shown simulation results for Exponential distribution using WMN-GA, WMN-TS, WMN-HC and WMN-SA, respectively. If we compare the results, the WMN-TS, WMN-HC and WMN-SA perform better than WMNGA for all radius of communication distances.

In Fig. 15, Fig. 16, Fig. 17 and Fig. 18 are shown simulation results for Weibull distribution using WMN-GA, WMN-TS, WMN-HC and WMN-SA, respectively. If we compare the results, the WMN-TS has a good performance for radius of communication distance less than $2 \times 2: 6 \times 6$, but for $2 \times 2: 8 \times 8$ the WMN-GA, WMN-HC and WMN-SA perform better.

\section{Conclusions}

In this paper, we deal with connectivity and coverage problem in WMNs. We used Friedman test to compare GA, TS, HC and SA. Then, we used the web interface to evaluate and compare the performance of the system for GA, TS, HC and SA for different distributions of mesh clients in terms of SGC and NCMC. From the simulation results, we conclude as follows.

- Using Friedman test we found out that GA, TS, HC and SA have difference in their performance.

- For Uniform distribution the WMN-HC and WMN-SA perform better than WMN-GA and WMN-TS. However, for radius of communication distance $2 \times 2: 8 \times 8$, the SGC of WMN-TS is better than other systems.

- For Normal distribution, for radius of communication distance $2 \times 2: 8 \times 8$, the WMNGA has the best performance. 
- For Exponential distribution, the WMN-HC and WMN-SA perform better than WMN-GA for all radius of communication distances.

- For Weibull distribution, the WMN-TS has a good performance for radius of communication distance less than $2 \times 2: 6 \times 6$, but for $2 \times 2: 8 \times 8$, the WMN-GA, WMN$\mathrm{HC}$ and WMN-SA perform better.

In the future work, we would like to make extensive simulations to evaluate the performance of WMN-GA and WMN-TS systems for different scenarios and parameters.

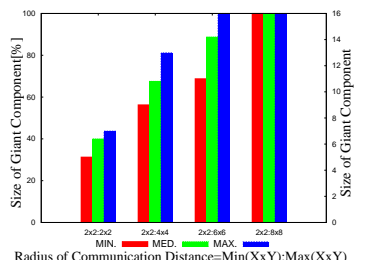

(a) SGC VS. radius of

Communication Distance.

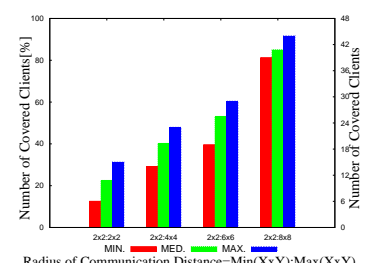

(b) NCMC Vs, radius of Communication Distance.

Figure 3 Simulation results of WMN-GA for Uniform distribution.

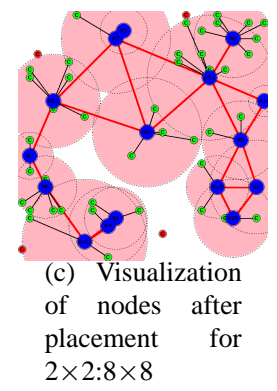

isualization $2 \times 2: 8 \times 8$
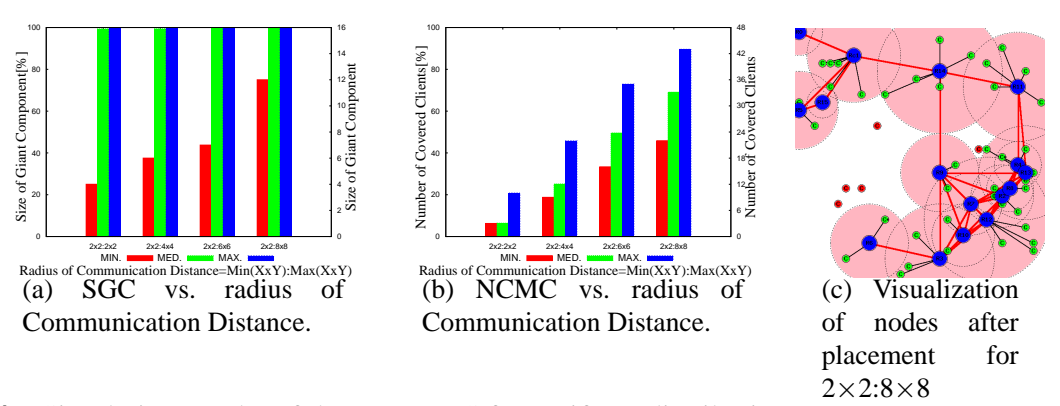

Figure 4 Simulation results of the WMN-TS for Uniform distribution.
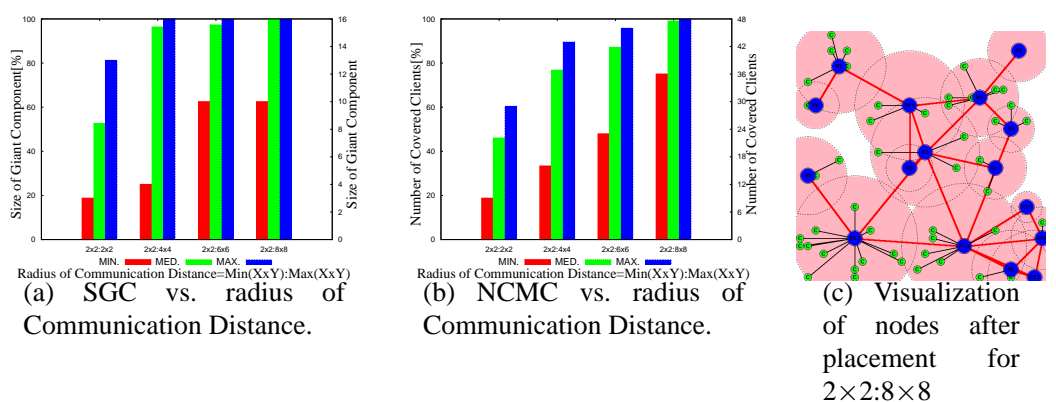

Figure 5 Simulation results of the WMN-HC for Uniform distribution. 


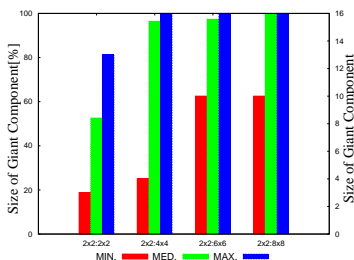

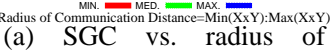

Communication Distance.

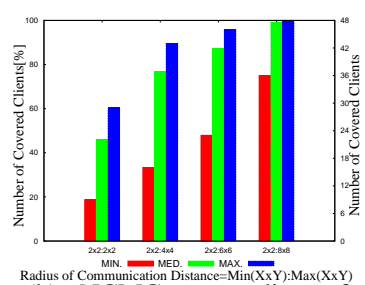

(b) NCMC vs. radius of

Communication Distance.

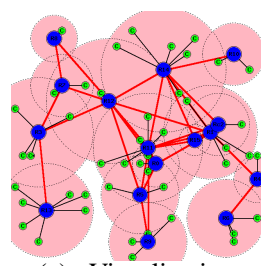

(c) Visualization of nodes after placement for $2 \times 2.8 \times 8$

Figure 6 Simulation results of the WMN-SA for Uniform distribution.

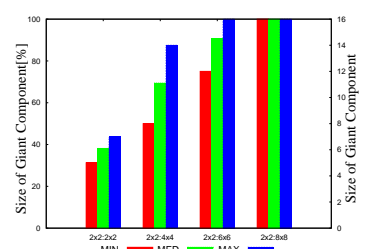

Radius of Communication Distance $=\operatorname{Min}(\mathrm{XXX} Y): \operatorname{Max}(\mathrm{XxY})$
(a) SGC VS. radius of

Communication Distance.

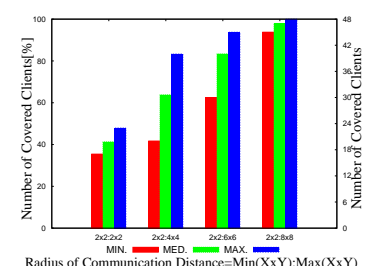

(b) NCMC Vs. radius of

Communication Distance.

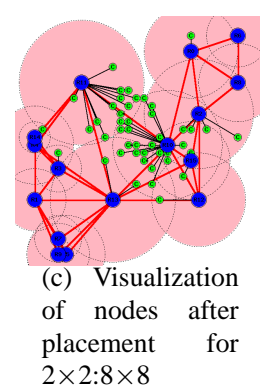

Figure 7 Simulation results of WMN-GA for Normal distribution.
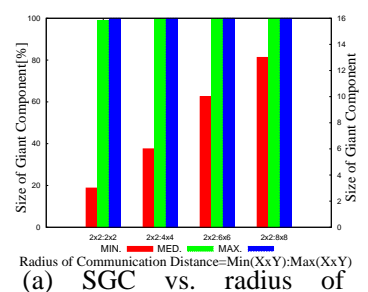

Communication Distance.

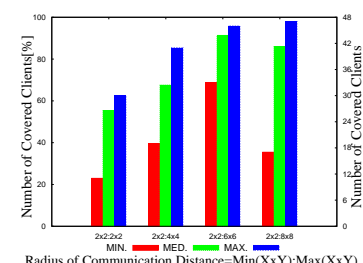

(b) NCMC Vs, radius of Communication Distance.

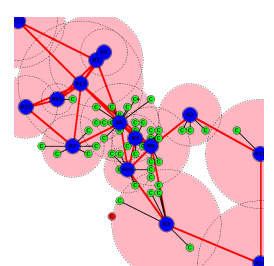

(c) Visualization of nodes after placement for $2 \times 2: 8 \times 8$

Figure 8 Simulation results of the WMN-TS for Normal distribution.

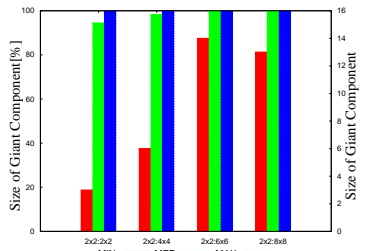

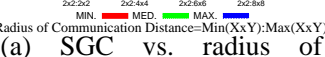

Communication Distance.

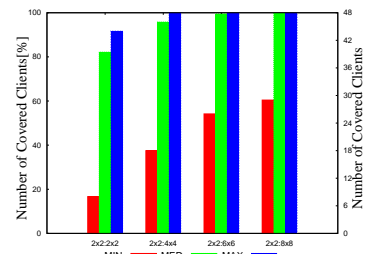

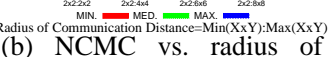

Communication Distance.

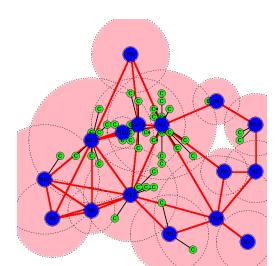

(c) Visualization of nodes after placement for $2 \times 2: 8 \times 8$

Figure 9 Simulation results of the WMN-HC for Normal distribution. 


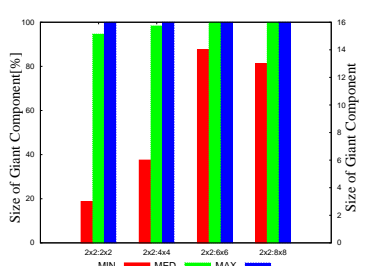

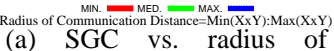

Communication Distance.

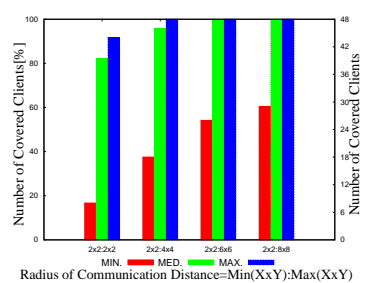

Radius of Cominunication Distance MAX Min(XXY):Max(XXY)
(b) NCMC Vs. radius of

Communication Distance.

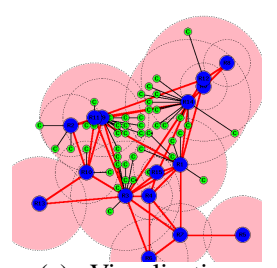

(c) Visualization of nodes after placement for $2 \times 2: 8 \times 8$

Figure 10 Simulation results of the WMN-SA for Normal distribution.

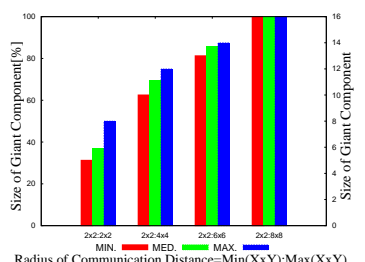

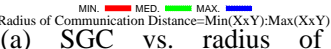

Communication Distance.

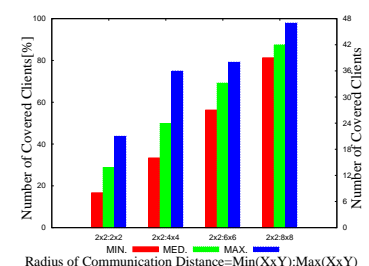

(b) NCMC Vs. radius of

Communication Distance.

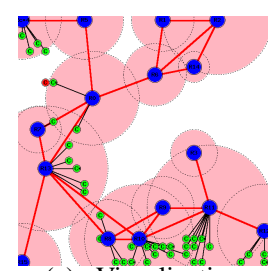

c) Visualization

of nodes after placement for $2 \times 2.8 \times 8$

Figure 11 Simulation results of WMN-GA for Exponential distribution.
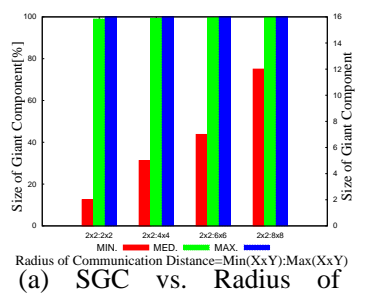

Communication Distance.

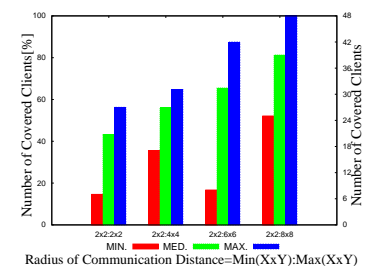

(b) NCMC vs. Radius of Communication Distance.

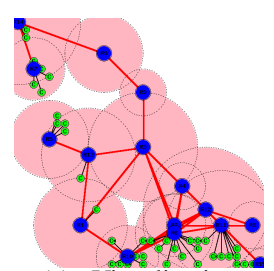

(c) Visualization of nodes after placement for $2 \times 2: 8 \times 8$

Figure 12 Simulation results of WMN-TS for Exponential distribution.

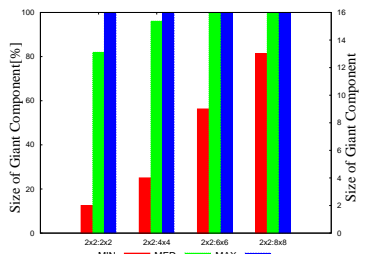

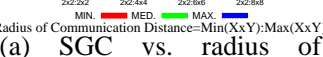
Communication Distance.

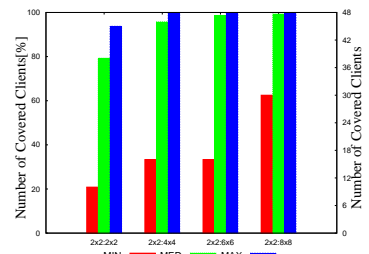

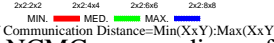

(b) NCMC VS. radius of Communication Distance.

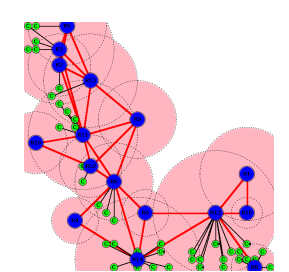

(c) Visualization of nodes after placement for $2 \times 2: 8 \times 8$

Figure 13 Simulation results of the WMN-HC for Exponential distribution. 
14

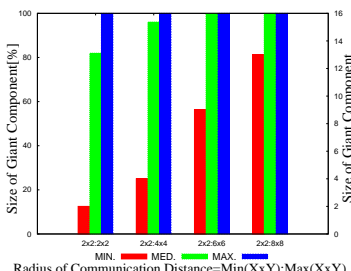

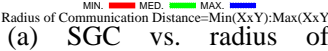

Communication Distance.

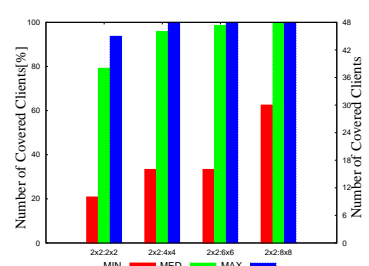

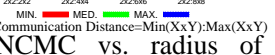

Communication Distance.

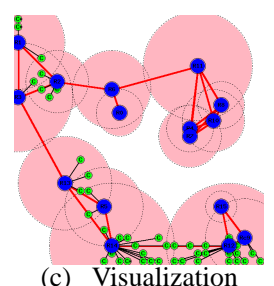

(c) Visualization

of nodes after placement for $2 \times 2: 8 \times 8$

Figure 14 Simulation results of the WMN-SA for Exponential distribution.
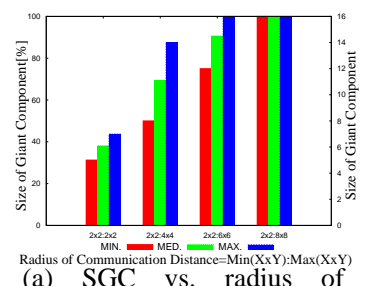

communication distance.

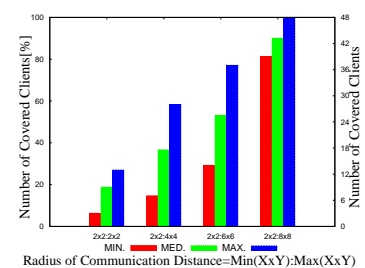

Radius of Communication Distance $=\operatorname{Min}(\mathrm{XXY} Y): \operatorname{Max}(\mathrm{XXY})$
(b) NCMC Vs. radius of

Communication Distance.

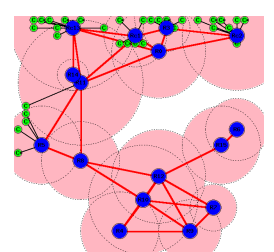

(c) Visualization of nodes after placement for $2 \times 2: 8 \times 8$

Figure 15 Simulation results of WMN-GA for Weibull distribution.
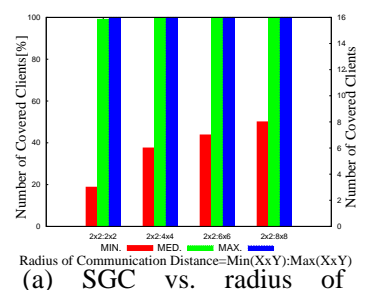

Communication Distance.

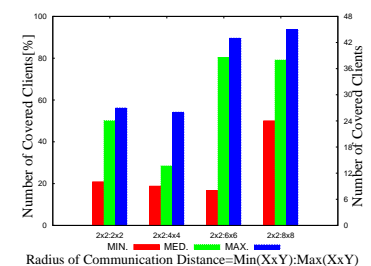

(b) NCMC Vs, radius of Communication Distance.

Figure 16 Simulation results of WMN-TS for Weibull distribution.

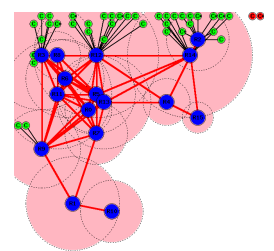

(c) Visualization of nodes after placement for $2 \times 2: 8 \times 8$

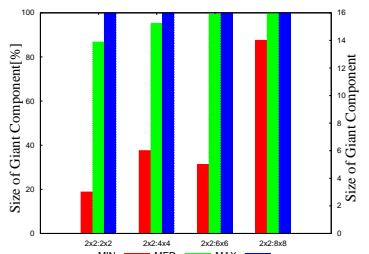

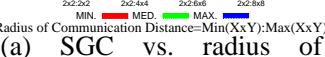

Communication Distance.

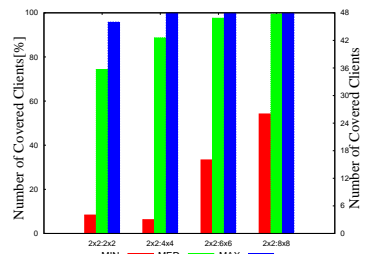

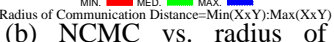
Communication Distance.

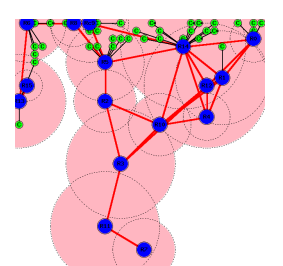

(c) Visualization of nodes after placement for $2 \times 2: 8 \times 8$

Figure 17 Simulation results of the WMN-HC for Weibull distribution. 


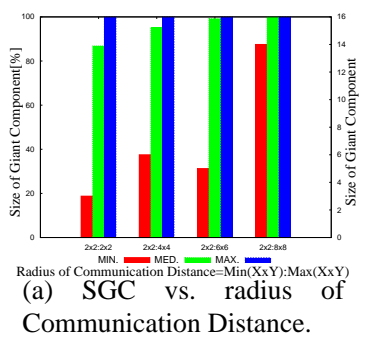

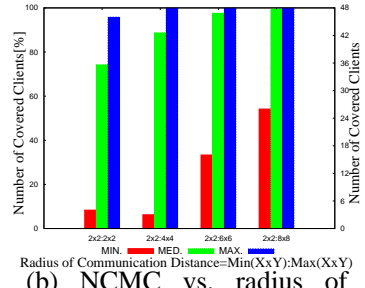

Communication Distance.

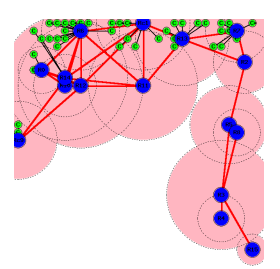

(c) Visualization of nodes after placement for $2 \times 2: 8 \times 8$

Figure 18 Simulation results of the WMN-SA for Weibull distribution.

\section{References}

O. Boyinbode, H. Le, M. Takizawa, "A Survey on Clustering Algorithms for Wireless Sensor Networks", International Journal of Space-Based and Situated Computing, Vol. 1, No. 2/3, pp. 130-136, 2011.

M. Puzar, T. Plagemann, "Data Sharing in Mobile Ad-hoc Networks - A Study of Replication and Performance in the MIDAS Data Space", International Journal of Space-Based and Situated Computing, Vol. 1, No. 2/3, pp. 137-150, 2011.

D. Martins, H. Guyennet, "Security in Wireless Sensor Networks: A Survey of Attacks and Countermeasures", International Journal of Space-Based and Situated Computing, Vol. 1, No. 2/3, pp. 151-162, 2011.

A. Koyama, Y. Sato, L. Barolli, "IEZRP: An Improved Enhanced Zone-based Routing Protocol for MANETs and its Performance Evaluation”, International Journal of Space-Based and Situated Computing, Vol. 1, No. 4, pp. 213-221, 2011.

M. Hiyama, E. Kulla, M. Ikeda, L. Barolli, "Evaluation of MANET Protocols for Different Indoor Environments: Results from a Real MANET Testbed", International Journal of Space-Based and Situated Computing, Vol. 2, No. 2, pp. 71-82, 2012.

M. Ikeda, "Analysis of Mobile Ad-hoc Network Routing Protocols using Shadowing Propagation Model”, International Journal of Space-Based and Situated Computing, Vol. 2, No. 3, pp. 139148, 2012.

E. Spaho, G. Mino, L. Barolli, F. Xhafa, "Goodput and PDR analysis of AODV, OLSR and DYMO protocols for vehicular networks using CAVENET", International Journal of Grid and Utility Computing, Vol. 2, No. 2, pp. 130-138, 2011.

A. Aikebaier, T. Enokido, M. Takizawa, "TMPR-scheme for Reliably Broadcast Messages Among Peer Processes", International Journal of Grid and Utility Computing, Vol. 2, No. 3, pp. 175182, 2011.

F. Xhafa, J. Kolodziej, L. Barolli, V. Kolici, R. Miho, M. Takizawa, "Hybrid Algorithms for Independent Batch Scheduling in Grids", International Journal of Web and Grid Services, Vol. 8, No. 2, pp. 134-152, 2012.

F. Xhafa, J. Sun, A. Barolli, A. Biberaj, L. Barolli, "Genetic Algorithms for Satellite Scheduling Problems", International Journal of Mobile Information Systems (MIS), IOS Press, Vol. 8, No. 4, pp. 351-377, 2012.

K. Goto, Y. Sasaki, T. Hara, S. Nishio, "Data Gathering using Mobile Agents for Reducing Traffic in Dense Mobile Wireless Sensor Networks", International Journal of Mobile Information Systems (MIS), IOS Press, Vol. 9, No. 4, pp. 295-314, 2013.

I. F. Akyildiz, X. Wang, and W. Wang, "Wireless Mesh Networks: A Survey", Computer Networks Vol. 47, No. 4, pp. 445-487, 2005. 
16.

J. B. Ernst, J. A. Brown, "Performance evaluation of mixed-bias scheduling schemes for wireless mesh networks", International Journal of Space-Based and Situated Computing, Vol. 3, No. 1, pp. 22-34, 2013.

S. N. Muthaiah and C. Rosenberg, "Single Gateway Placement in Wireless Mesh Networks", In Proc. of 8th International IEEE Symposium on Computer Networks, Turkey, pp. 4754-4759, 2008.

M. Tang, "Gateways Placement in Backbone Wireless Mesh Networks", International Journal of Communications, Network and System Sciences, Vol. 2, No.1, pp. 45-50, 2009.

A. Antony Franklin and C. Siva Ram Murthy, "Node Placement Algorithm for Deployment of TwoTier Wireless Mesh Networks", In Proc. of IEEE GLOBECOM-2007, Washington, USA, pp. 4823-4827, 2007.

T. Vanhatupa, M. Hännikäinen and T.D. Hämäläinen, "Genetic Algorithm to Optimize Node Placement and Configuration for WLAN Planning", In Proc. of 4th International Symposium on Wireless Communication Systems, pp. 612-616, 2007.

M. R. Garey and D. S. Johnson, "Computers and Intractability -A Guide to the Theory of NPCompleteness", Freeman, San Francisco, 1979.

A. Lim, B. Rodrigues, F. Wang and Zh. Xua, " $k$-Center Problems with Minimum Coverage", Theoretical Computer Science, Vol. 332, No. 1-3, pp. 1-17, 2005.

J. Wang, B. Xie, K. Cai and D. P. Agrawal, "Efficient Mesh Router Placement in Wireless Mesh Networks", MASS, Pisa, Italy, pp. 9-11, 2007.

X. Yao, "An Empirical Study of Genetic Operators in Genetic Algorithms", In 19th EUROMICRO Symposium on Microprocessing and Microprogramming on Open System Design: Hardware, Software and Applications (Barcelona, Spain), Elsevier Science Publishers, pp. 707-714, 1993.

J. Denzinger and J. Kidney, "Evaluating Different Genetic Operators in the Testing for Unwanted Emergent Behavior Using Evolutionary Learning of Behavior", In Proc. of the IEEE/WIC/ACM International Conference on Intelligent Agent Technology, IEEE Computer Society, Washington, DC, pp. 23-29, 2006.

M. O. Odetayo, "Empirical Study of the Interdependencies of Genetic Algorithm Parameters", 23rd EUROMICRO Conference, New Frontiers of Information Technology, pp. 639, 1997.

F. Xhafa, B. Duran, A. Abraham, K. Dahal, "Tuning Struggle Strategy in Genetic Algorithms for Scheduling in Computational Grids", Neural Network World, Vol. 18, No. 3, 209-225, 2008.

F. Xhafa, L. Barolli, and A. Durresi, "An Experimental Study on Genetic Algorithms for Resource Allocation on Grid Systems", Journal of Interconnection Networks, Vol. 8, No. 4, pp. 427-443, 2007.

F. Xhafa, C. Sanchez, L. Barolli, "Ad Hoc and Neighborhood Search Methods for Placement of Mesh Routers in Wireless Mesh Networks", In Proc. of ICDCS Workshops of the IEEE 29th International Conference on Distributed Computing Systems (ICDCS-2009), pp. 400-405, 2009.

T. Oda, A. Barolli, E. Spaho, F. Xhafa, L. Barolli, M. Takizawa, "Evaluation of WMN-GA for Different Mutation Operators", International Journal of Space-Based and Situated Computing (IJSSC), Inderscience, Vol. 2, No. 3, pp. 149-157, 2012.

F. Glover, "Future Paths for Integer Programming and Links to Artificial Intelligence", Computers and Op. Res., pp. 533-549, 1986.

S. Kirkpatrik, C. D. Gelatt and M. P. Vecchi, "Optimization by Simulated Annealing", Journal of Science, Vol. 220, pp. 671-680, 1983.

F. Xhafa, C. Sanchez, L. Barolli, R. Miho, "An AnnealingApproach to Router Nodes Placement Problem in Wireless Mesh Networks", In Proc. of CISIS-2010, pp.245-252, 2010.

F. Xhafa, A. Bravo, A. Barolli, M. Takizawa, "An Interface for Simulating Node Placement in Wireless Mesh Networks", In Proc. of 15th International Conference on Network-Based Information Systems (NBiS-2012), pp. 326-333, 2012. 
M. Friedman, "The Use of Ranks to Avoid the Assumption of Normality Implicit in the Analysis of Variance”, Journal of the American Statistical Association, Vol. 32, No. 200, pp. 675-701, 1937. 